\title{
The chemical evolution of dwarf starburst galaxies
}

\author{
Francesca Matteucci \\ Dipartimento di Astronomia, Universitá di Trieste, \\ Via G.B. Tiepolo 11, I-34100 Trieste, and SISSA/ISAS, Trieste, Italia
}

\author{
Annibale D'Ercole \\ Osservatorio Astronomico di Bologna, I-44100 Bologna, Italia
}

\begin{abstract}
We will review the most popular models for the chemical evolution of some starburst galaxies, in particular dwarf irregular galaxies. These galaxies are relatively simple and unevolved objects with low metallicities and large gas contents, suggesting that they are either young or have undergone discontinuous star formation activity. Some dwarf irregulars are starburst galaxies currently experiencing an intense star formation event and they are known as blue compact galaxies or extragalactic $\mathrm{H}$ II regions. We will discuss the effects of the presence of dark matter halos together with stellar energetics (stellar winds and supernovae) on the development of a galactic wind in these systems. Particular emphasis will be given to the role of massive stars in driving the thermal and chemical evolution of the gas, in particular to type II supernovae. A comparison between different model predictions for abundances and abundance ratios will be used to impose constraints on the star formation history and on the amount of dark matter, which we found to be extremely important in these systems.
\end{abstract}

\section{Introduction}

The metallicity, gas content and colors of dwarf irregular galaxies indicate that they are either young or have experienced a sporadic star formation activity (bursts). For most of these galaxies the second hypothesis is the most likely to be true although a different star formation regime can probably be envisaged for dwarf irregulars (DIGs) and blue compact galaxies (BCGs). In particular, DIGs should have almost a continuous (gasping) star formation regime (Tosi et al. 1992), whereas the BCGs should experience few bursts of star formation. However, one cannot exclude that some of these galaxies are truly young objects in the sense that they have started forming stars only recently. The best known case of this type is the BCG IZw 18, which shows the lowest metal abundance known in local galaxies. Its oxygen abundance derived from $\mathrm{H}$ II regions is 1/30 of the solar value $\left(\sim 1.75 \times 10^{-5}\right.$ by number $)$. These galaxies are also likely to have undergone galactic winds due to their relatively low potential well. Another striking feature of these objects is the large spread in their properties especially in the mass-metallicity and metallicity-metallicity relations.

In the last few years a great deal of theoretical models for DIGs and BCGs appeared in the literature. By means of analytical models, Matteucci \& Chiosi (1983) first studied the effects of galactic winds on the chemical evolution of these galaxies, and suggested, among other hypotheses, that galactic winds of variable intensity could explain the observed spread in the physical properties of these 
galaxies. In the following years, by means of numerical models, Matteucci \& Tosi (1985), Pilyugin (1992, 1993) and Marconi et al. (1994), studied the effects of galactic winds on the chemical evolution of such galaxies and confirmed the previous results. In these papers the possibility of metal enriched winds was also considered and favoured relative to the normal galactic winds. This metal enriched wind hypothesis allowed them to explain the observed He-abundance vs. metal-abundance ratios, which show a substantial increase of He relative to metals in these objects (Pagel et al. 1992). However, none of these studies had taken into account either the energy injected by supernovae and stellar winds into the ISM or the presence of dark matter halos which are of fundamental importance for the development of a galactic wind. The galactic winds were just assumed to occur every time the starburst was active.

Dark matter in dwarf irregular galaxies seems to be quite important as shown by recent data indicating an increasing amount of dark matter with decreasing luminosity (Skillman 1996), and therefore it cannot be ignored in chemical evolution models. Kumai \& Tosa (1992) also explored the possibility of dark matter in these galaxies in a very simple way, and suggested that various amounts of dark matter could explain the observed spread in the $Z$ vs. $\log \left(M_{\text {gas }} / M_{\text {tot }}\right)$ relation.

The energetics of supernovae and stellar winds and their interaction with the ISM, is a difficult problem, still not quite understood. The majority of models of chemical evolution taking into account the stellar energetics in connection to the occurrence of galactic winds, are those developed to study the chemical and photometric evolution of elliptical galaxies (Arimoto \& Yoshii 1987; Matteucci \& Tornambé 1987). Recently, Bradamante et al. (1998) presented a model of chemical evolution for dwarf starburst galaxies which takes into account both the stellar energetics and the presence of dark matter as factors affecting the evolution of these objects. In this model the development of a galactic wind is calculated in detail and the existence of metal enriched (or differential) winds is also taken into account. By enriched wind we intend a wind carrying out only some heavy elements, in particular those produced by type II supernovae which are the predominant supernovae during a starburst (Marconi et al. 1994).

Therefore under this assumption, elements such as nitrogen and helium (although at solar metallicities also massive stars can be important He producers), which are mainly produced and restored by low and intermediate mass stars through stellar winds, will not leave the galaxy since their progenitor stars are likely to die in a different place from the birthplace whereas oxygen and the other $\alpha$-elements, ejected during type II supernova explosions, are likely to be ejected outside the star forming region and perhaps outside the galaxy through the galactic wind triggered by the energy injection from their progenitors. Iron will only in part leave the star forming region since only a fraction of this element is produced by SNe II, whereas the bulk of it comes from SNe Ia. It should be again said that the differential wind assumption has proven to be the most viable solution to solve the helium problem in blue compact galaxies (Pilyugin 1993; Marconi et al. 1994).

In the following we will describe the results of the Marconi et al. (1994) model and those of the Bradamante et al. (1998) (where a detailed description can be found) model and compare them with observations. 


\section{The Marconi et al. model}

This model assumes that the star formation in BCGs proceeds in short but intense bursts of star formation and the number of bursts varies from 1 to 15 with an average duration of $100 \mathrm{Myr}$. The novelty, relative to previous models (e.g., Matteucci \& Tosi 1995), is the fact that detailed nucleosynthesis from type II and Ib supernovae, assumed to be the result of the explosion of WR stars, and type Ia supernovae is taken into account. In particular, the type Ia SN rate is calculated as described in Matteucci \& Greggio (1986), by assuming that the progenitor of these $\mathrm{SNe}$ are binary systems made of a $\mathrm{C}-\mathrm{O}$ white dwarf plus a red giant star. Galactic winds are assumed to occur each time a starburst is active and the rate of mass loss from the galaxy is proportional to the star formation rate. Therefore in this framework the gas is lost continuously from the galaxy when star formation is active but suddenly stops during the interburst phases. In this model galactic winds are normal and differential. In the second case it is assumed that only the products of type II (and Ib) SNe are ejected from the galaxy. This assumption is likely to be true as recently shown by dynamical models of starbursts (Mac Low \& Ferrara 1998; Brighenti \& D'Ercole, these Proceedings). From the observational point of view, galactic winds are observed in these objects, as suggested by the inferred velocities of outflowing material compared to the estimated escape velocities (Meurer et al. 1992; Papaderos et al. 1994; Lequeux et al. 1995; Martin 1996; Kunth et al. 1998). The evolution of the abundances of several specific elements $(\mathrm{H}, \mathrm{He}, \mathrm{C}, \mathrm{N}, \mathrm{O}, \mathrm{Fe})$ is followed in detail by relaxing the instantaneous recycling approximation and adopting the most up-to-date nucleosynthesis prescriptions. Marconi et al. concluded that enriched galactic winds are to be preferred to normal winds since they can explain the large growth of He relative to metals observed in these objects $\left(\frac{\Delta(\mathrm{He} / \mathrm{H})}{\Delta(\mathrm{O} / \mathrm{H})}=58\right.$ in number, Pagel et al. 1992). They also concluded that in starburst galaxies the SN II are dominating the chemical evolution of the gas. As a consequence of this, the $[\alpha / \mathrm{Fe}]>0$ in these objects if the number of bursts is limited. Finally, they suggested that the total number of bursts should not exceed ten.

\section{The Bradamante et al. model}

The Bradamante et al. (1988) model is substantially similar to the Marconi et al. model except that the development of a galactic wind is studied in detail by computing the binding energy and the thermal energy of the gas at any time. The thermal energy is calculated by assuming that either massive stars through stellar winds or supernovae inject energy into the ISM. From the theoretical point of view it is extremely difficult to estimate the right amount of energy which is transferred from supernovae and massive stars into ISM. Normally, supernovae are treated as isolated and so is their energy contribution. In reality, supernovae (of type II) instead occur in groups and the energy released and transferred into the ISM is much greater when their remnants overlap. Therefore, a correct treatment should take into account the filling factor of the gas. 


\subsection{Stellar energetics}

In this model the filling factor of the gas is not taken into account and it is assumed that galaxies develop galactic winds when the gas thermal energy $E_{g}^{t h}(t)$, exceeds its binding energy $E_{g}^{b}(t)$, i.e. when:

$$
E_{g}^{t h}(t) \geq E_{g}^{b}(t)
$$

The energetics of the ISM is considered in the following way:

$$
E_{g}^{t h}(t)=E_{S N}^{t h}(t)+E_{s w}^{t h}(t)
$$

where the energy fraction deposited in the gas by stellar winds from massive stars is $E_{s w}^{t h}(t)$, and by supernova explosions is $E_{S N}^{t h}(t)$. The supernova contribution is given by:

$$
E_{S N}^{t h}(t)=E_{S N I I}^{t h}(t)+E_{S N I a}^{t h}(t)
$$

where:

$$
\begin{gathered}
E_{S N I I}^{t h}=\int_{0}^{t} \epsilon_{S N} R_{S N I I}\left(t^{\prime}\right) d t^{\prime} \\
E_{S N I a}^{t h}=\int_{0}^{t} \epsilon_{S N} R_{S N I a}\left(t^{\prime}\right) d t^{\prime} \\
E_{s w}^{t h}(t)=\int_{0}^{t} \int_{12}^{m_{u p}} \phi(m) \psi\left(t^{\prime}\right) \epsilon_{w} d m d t^{\prime}
\end{gathered}
$$

$R_{S N I I}(t)$ and $R_{S N I a}(t)$ are the rates of supernova (II, Ib and Ia) explosion, $\phi(m)$ is the IMF and $\psi(t)$ is the star formation rate. The type Ia and II SN rates are calculated according to Matteucci \& Greggio (1986). The energy injected and effectively thermalized into the ISM from supernova explosions $\left(\epsilon_{S N}\right)$ and stellar winds from massive stars $\left(\epsilon_{w}\right)$, are given by the following formulas:

$$
\begin{gathered}
\epsilon_{S N}=\eta_{S N} E_{0} \\
\epsilon_{w}=\eta_{w} E_{w}
\end{gathered}
$$

where $E_{0}$ is the total energy released by a supernova explosion, $E_{w}$ is the energy injected into the ISM by a typical massive star through stellar winds during all its lifetime, and $\eta_{S N}$ and $\eta_{w}$ are the efficiencies of energy transfer from supernova and stellar winds into the ISM, respectively. In order to choose the right efficiencies of energy injection for massive stars and supernovae they referred to an ideal case characterized by a uniform ISM, and no interaction with other supernova explosions or interstellar clouds. When a supernova explodes the stellar material is violently ejected into the ISM and its expansion is gradually slowed down by the ISM. The energy released by the supernova explosion is assumed to be $E_{0}=10^{51} \mathrm{erg}$ but much of it is radiated away during the snowplow phase 
following the Sedov expansion. The energy effectively transferred and thermalized into the ISM is given by eq. (13) and depends on the assumed value for $\eta_{S N}$. The main parameters used to derive $\eta_{S N}$ are: the initial blast wave energy $E_{51}=E_{0} /\left(10^{51} \mathrm{erg}\right)$, the interstellar gas density $n_{0}$, and the isothermal sound speed in the ISM $c_{0,6}=c_{0} /\left(10^{6} \mathrm{~cm} \mathrm{~s}^{-1}\right)$.

For typical values of these parameters $E_{51}=1, n_{0}=1 \mathrm{~cm}^{-3}$ and $c_{0,6} \simeq 1$ and assuming that the residual energy of the $\mathrm{SN}$ remnant goes effectively into the ISM only when $v_{\text {shell }}=c_{0,6}$ one obtains the following range of possible values for $\eta_{S N}$ :

$$
\eta_{S N}=0.007 \div 0.13
$$

In particular, they have adopted an intermediate value of $\eta_{S N}=0.03$ assumed to be the typical SN energy transfer efficiency. It is worth noting that if the SN remnants are indeed overlapping the amount of energy transferred into the ISM can be much larger. On the other hand, in a more realistic situation for the ISM (e.g., not homogeneous) the loss of energy from the remnant could be larger than estimated.

The energy injected into the ISM by a typical massive star through stellar winds during all its lifetime is estimated to be:

$$
E_{w}=L_{w} t_{M S}
$$

where $t_{M S}$ is the time the star spends in Main Sequence and $L_{w}$ is the stellar wind luminosity. By adopting the energy conserving solution from Weaver et al. (1977) they obtained:

$$
\eta_{w}=0.3\left(L_{36}\right)^{2 / 3} n_{0}^{-1 / 2} c_{0,6}^{-5 / 2}
$$

if one assumes that a $20 M_{\odot}$ star is a typical massive star contributing to the stellar winds then $L_{36}=L_{w} /\left(10^{36} \mathrm{erg} \mathrm{s}^{-1}\right)=0.03, t_{M S}=7.9 \times 10^{6} \mathrm{yr}$ with $n_{0}=1 \mathrm{~cm}^{-3}$ and $c_{0,6}=1$, one obtains:

$$
E_{w} \simeq 10^{49} \text { erg and } \eta_{w}=0.03
$$

In summary, the following were adopted:

$$
\epsilon_{S N}=0.03 \cdot 10^{51} \mathrm{erg}
$$

and

$$
\epsilon_{w}=0.03 \cdot 10^{49} \mathrm{erg}
$$

\subsection{Dark matter}

In the Bradamante et al. model dark matter is also taken into account and it influences the calculation of the potential energy. In particular, they computed the binding energy of interstellar gas, $E_{g}^{b}(t)$, in the following way:

$$
E_{g}^{b}=W_{L}(t)+W_{L D}(t)
$$


The two terms on the right of the equation take into account the gravitational interaction between the gas mass $M_{g}(t)$, and the total luminous mass of the galaxy $M_{L}(t)$, and between the gas mass and the dark matter $M_{d}$ :

$$
\begin{aligned}
& W_{L}(t)=-0.5 G \frac{M_{g}(t) M_{L}(t)}{r_{L}} \\
& W_{L D}(t)=-G \tilde{w}_{L D} \frac{M_{g}(t) M_{d}}{r_{L}}
\end{aligned}
$$

where $\quad \tilde{w}_{L D} \simeq \frac{1}{2 \pi} S[1+1.37 S]$.

$G$ is the gravitational constant, and $S=r_{L} / r_{d}$ is the ratio between the effective radius of luminous matter and the effective radius of dark matter. These equations are taken from Bertin et al. (1991) and are valid for $S$ defined in the range $0.10 \div 0.45$.

\subsection{Stellar nucleosynthesis}

The adopted nucleosynthesis prescriptions are:

- low and intermediate mass stars: the Renzini \& Voli (1981) prescriptions, their case $\alpha=1.5 \eta=0.33$ and $M_{u p}=8 \mathrm{M}_{\odot}$;

- massive stars: the prescriptions from Woosley(1987) and Woosley \& Weaver (1995); and

- SNIa: the Nomoto et al. (1984) nucleosynthesis calculations, their model W7.

The nucleosynthesis of nitrogen is still quite uncertain and deserves some discussion. The majority of $\mathrm{N}$ originates from low and intermediate mass stars although some $\mathrm{N}$ is also contributed by massive stars via stellar winds.

Nitrogen is normally a secondary element in the sense that it is produced from the $\mathrm{O}$ and $\mathrm{C}$ originally present in the star but in AGB stars $\mathrm{N}$ can be produced also as a primary element although the amount of this primary $\mathrm{N}$ depends crucially on the occurrence of the third dredge-up.

If $3^{\text {rd }}$ dredge-up does not occur, primary $\mathrm{N}$ should drop to a few percent of the amount predicted by Renzini \& Voli (1981). Recent nucleosynthesis calculations in AGB stars (van den Hoek \& Groenewegen 1997) predict a smaller amount of $\mathrm{N}$ than the yields of Renzini \& Voli but the proportions primary/secondary are roughly the same. The primary/secondary origin of $\mathrm{N}$ in massive stars is still not clear. Matteucci (1986) suggested the possibility of primary $\mathrm{N}$ in massive stars and recent calculations including stellar rotation by Maeder (these Proceedings) seem to confirm this possibility.

The nucleosynthesis of $\mathrm{He}$ is also rather uncertain. Calculations by Maeder (1992) predict large amounts of $\mathrm{He}$ to be restored by massive stars through stellar winds, but only for stars of high metal content (at least solar) so that these predictions do not apply to BCGs, DIGs and low metallicity high red-shift objects in general. 


\subsection{Some model results}

Several models were computed by assuming $M_{l u m}=10^{9} \mathrm{M}_{\odot}$, a different number of bursts of duration of $60 \mathrm{Myr}$ which is short enough to prevent the galaxy from blowing-up during a starbursts and long enough to guarantee the development of a galactic wind. The amount of dark matter was also fixed on the basis of the same criterion and it implies a ratio $R=M_{\text {dark }} / M_{\text {lum }}=10$ and $S=0.3$.

The star formation rate relative to an element $i$ is:

$$
S F R=\Gamma G(t) X_{i}(t)
$$

where $G_{i}(t)=G(t) X_{i}(t)$ is the fractional mass of the element $i$ present in the gas and $X_{i}$ is its abundance by mass and $\Gamma$ is the star formation efficiency. The galactic wind in this model is not strictly occurring during the starburst since

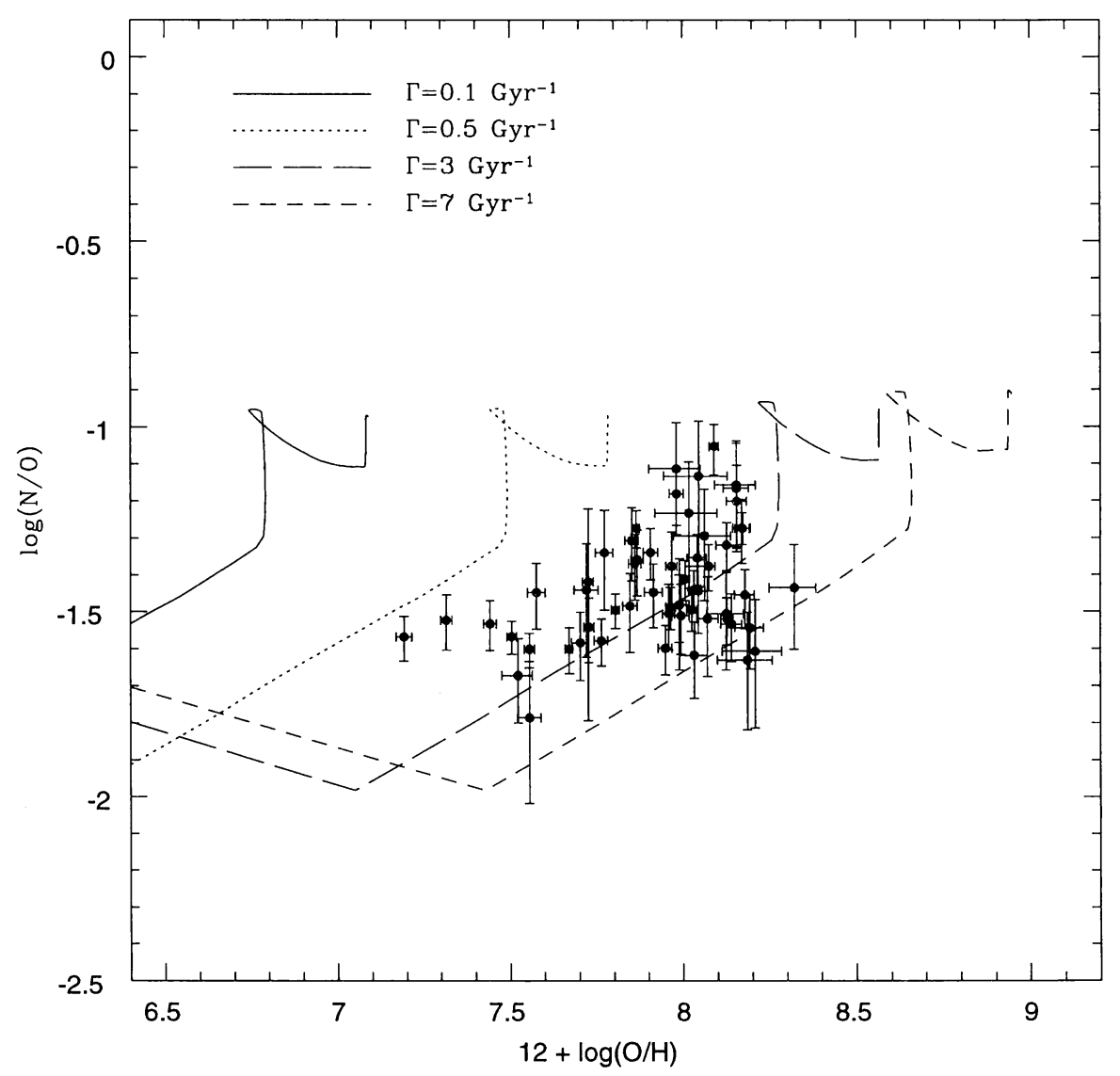

Figure 1. Predicted and observed $\log (\mathrm{N} / \mathrm{O})$ vs. $12+\log (\mathrm{O} / \mathrm{H})$. The model is a two-burst model. Each burst lasts for $0.06 \mathrm{Gyr}, R=10, S=0.3$, Salpeter IMF. The data are from Kobulnicky \& Skillman (1996). 


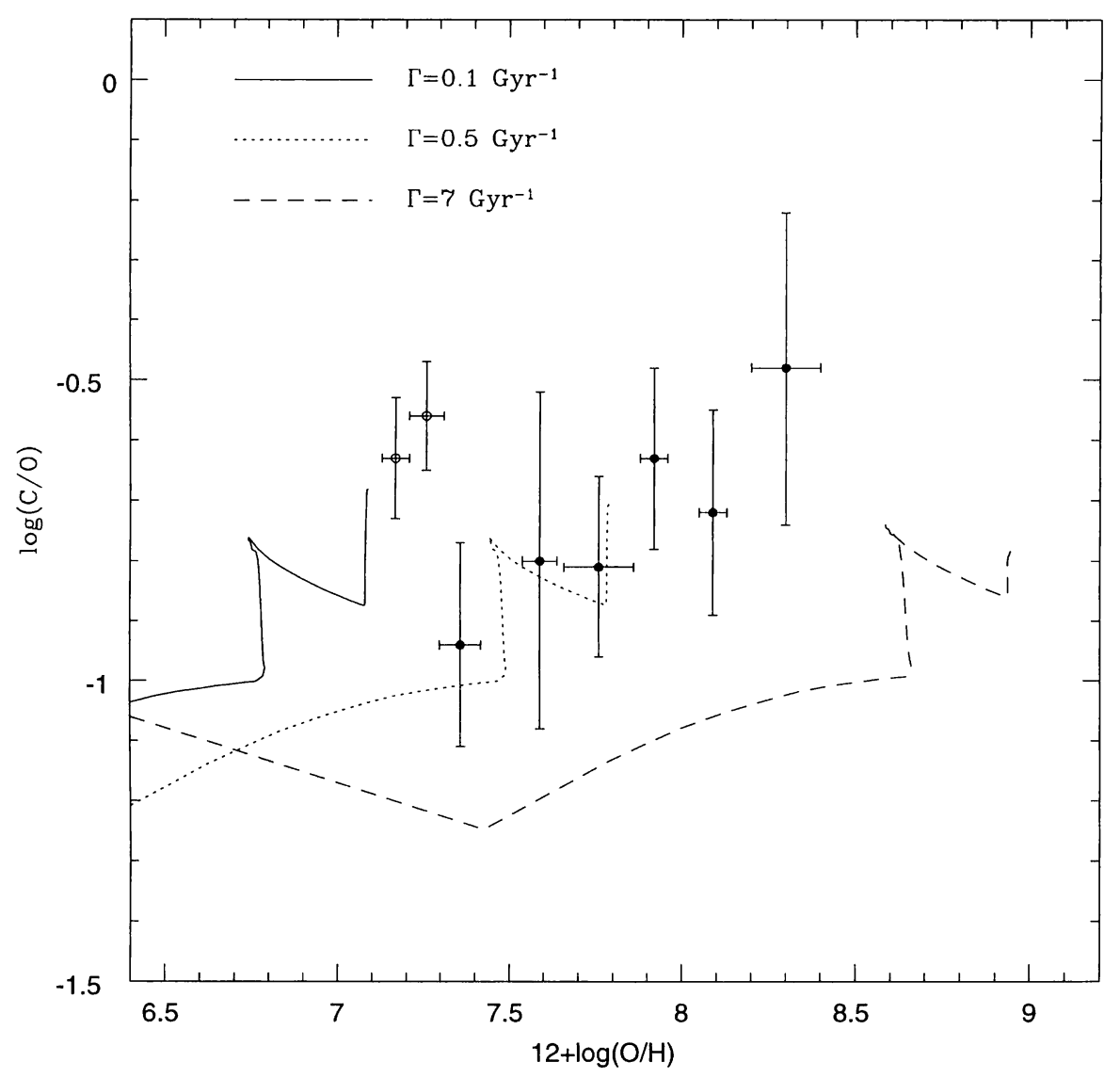

Figure 2. Predicted and observed $\log (\mathrm{C} / \mathrm{O})$ vs. $12+\log (\mathrm{O} / \mathrm{H})$. The model is a two-burst model. Each burst lasts for $0.06 \mathrm{Gyr}, R=10, S=0.3$, Salpeter IMF. The data are from Garnett et al. (1997) (open circles) and Garnett et al. (1995) (filled circles).

it depends on the thermal energy of gas and is influenced also by the energy released by type Ia SNe which continue to explode also during the interburst periods (see Marconi et al. 1994). The rate at which the fractional mass of an element $i$ is lost from the galaxy is:

$$
W R=w_{i} G(t) X_{i}(t)
$$

where $w_{i}$ is the efficiency of the wind rate for a specific element $i$. Under the assumption of enriched winds, as described before, $w_{i} \neq 0$ only for the elements contributed by type II SNe, mainly the $\alpha$ elements. The model was tested to reproduce the observed $\log (\mathrm{N} / \mathrm{O})$ vs. $12+\log (\mathrm{O} / \mathrm{H}), \log (\mathrm{C} / \mathrm{O})$ vs. $12+\log (\mathrm{C} / \mathrm{H})$ and $[\mathrm{O} / \mathrm{Fe}]$ vs. $[\mathrm{Fe} / \mathrm{H}]$. The data and the model predictions for a Salpeter $(1955)$ 


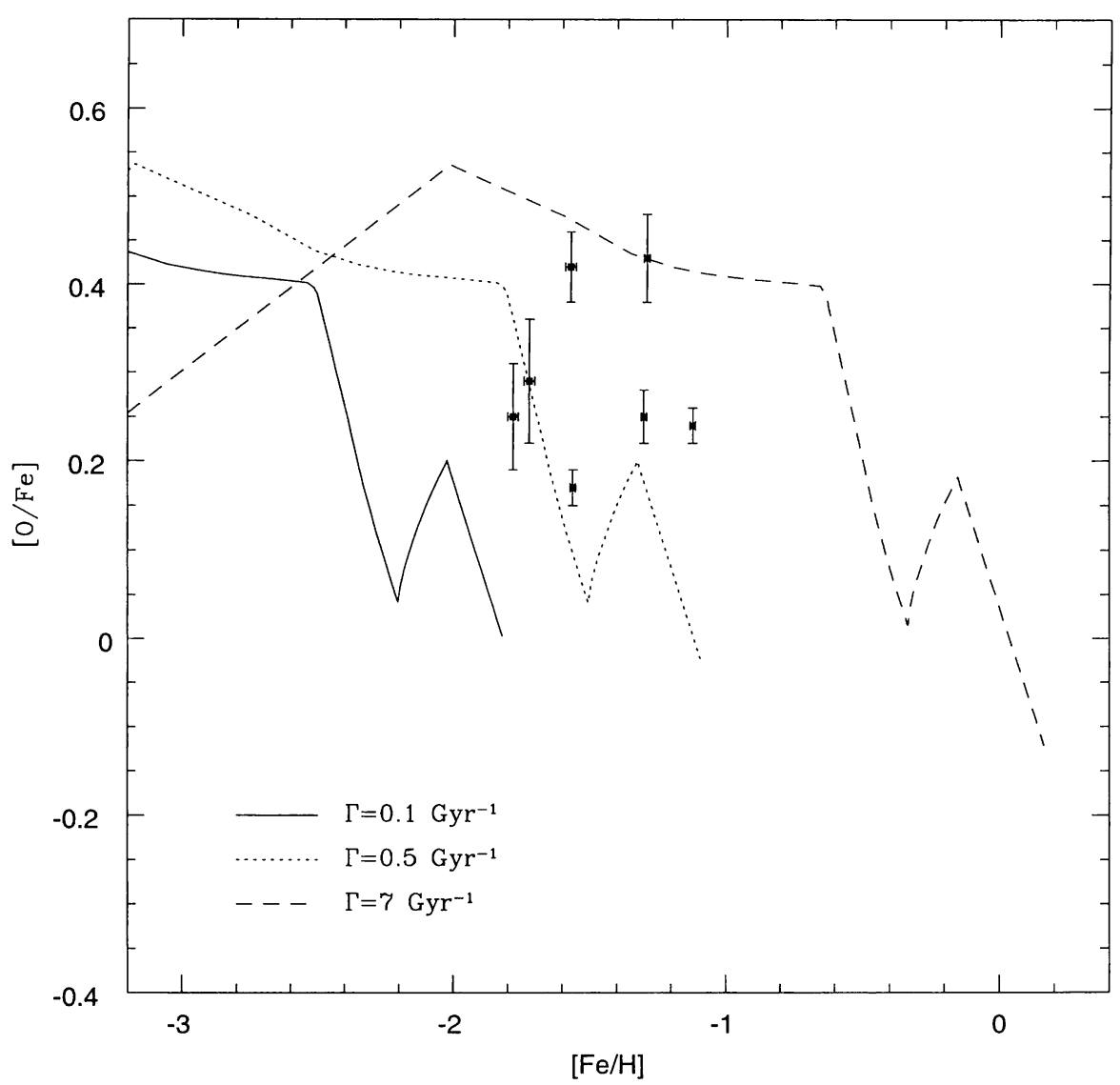

Figure 3. Predicted and observed $[\mathrm{O} / \mathrm{Fe}]$ vs. $[\mathrm{Fe} / \mathrm{H}]$. The model is a twoburst model. Each burst lasts for $0.06 \mathrm{Gyr}, R=10, S=0.3$, Salpeter IMF. The data are from Thuan et al. (1995).

IMF and different star formation efficiencies $\Gamma$ are shown in the Figures 1, 2 and 3.

As one can see, the parameter $\Gamma$ rather than the number of bursts, which would move the abundance ratios along the same line, is fundamental in explaining the observed spread. In particular, for a two-burst model the efficiency of star formation should vary from $\Gamma=0.1 \mathrm{Gyr}^{-1}$ to $\Gamma=7 \mathrm{Gyr}^{-1}$. Also evident in the figures is the sawtooth behaviour of the abundance ratios due to the fact that during the bursts all elements are produced whereas during the interburst periods only the elements originating from long living stars are produced (for example $\mathrm{N}$ and $\mathrm{Fe}$ ). Therefore, part of the observed spread could be just due to the fact that some galaxies are observed during the interburst period and others during the burst period. 


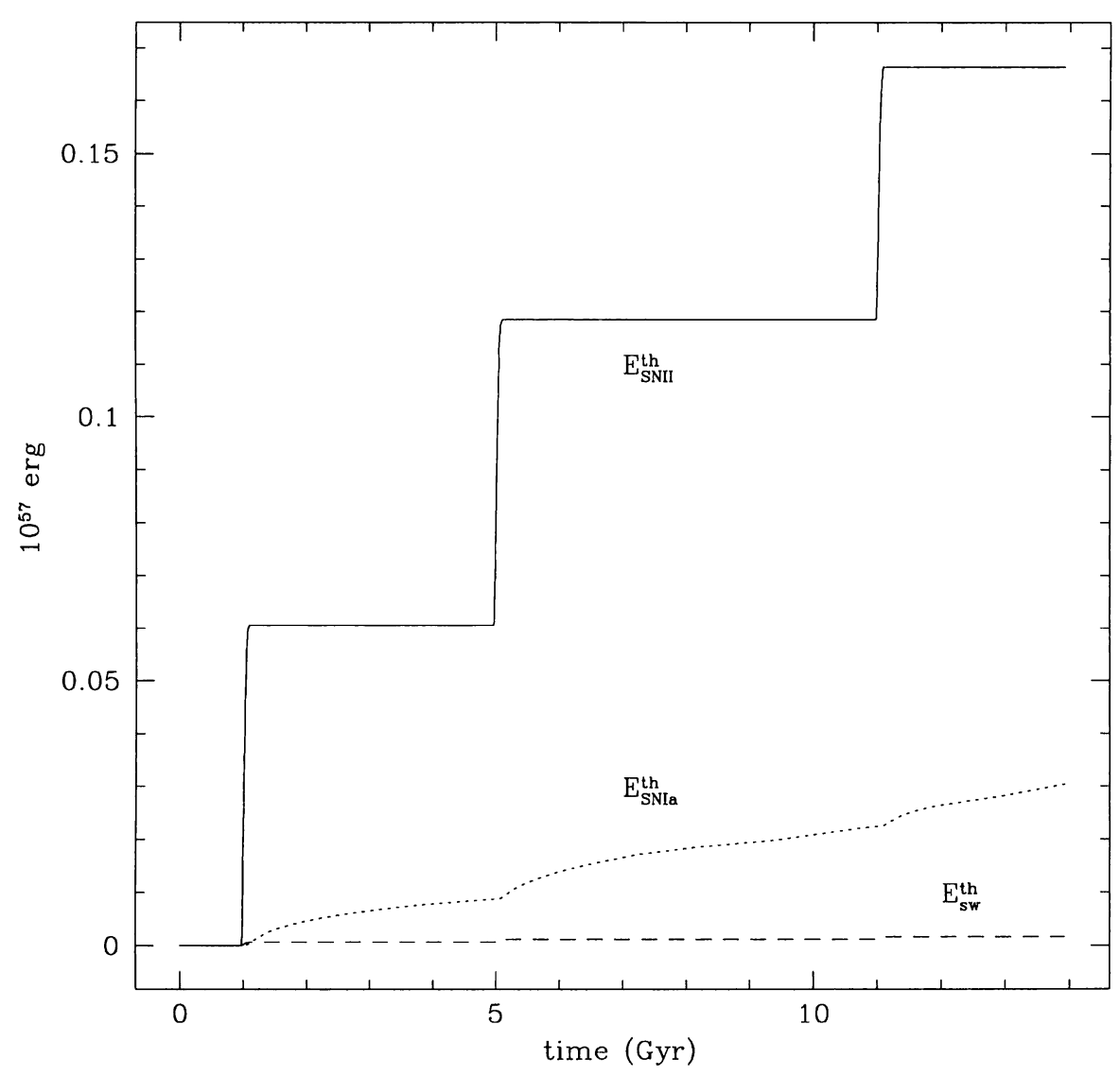

Figure 4. Predicted thermal energies of the gas as due to different energy sources (SNe II, SNe Ia, massive stars). The energies are given in units of $10^{57}$ erg. The model is a three-burst model. Each burst lasts for $0.06 \mathrm{Gyr}, R=10$, $S=0.3$, Salpeter IMF .

Concerning the dark matter, Bradamante et al. (1998) concluded that it is necessary to assume a massive dark halo in order to avoid the galaxy from blowing out. In fact, the star formation rate is fixed by reproducing the abundances and the abundance ratios in these objects, and unless the assumed efficiencies of energy tranfer from stars to ISM are too high, which is unlikely, the only solution is to include a dark matter halo which deepens the potential well. In particular, they predict that the ratio $R$ should vary from 1 to 10 to reproduce the majority of these galaxies for a maximum number of ten bursts.

Finally, in Figure 4 the comparison between the thermal energy of gas as due to SNeII (and Ib), SNe Ia and stellar winds from massive stars is shown. From the figure it is evident that SNeII are clearly dominating the energetics 
of the ISM in starburst galaxies and that the contribution of stellar winds is negligible once the first supernovae have started exploding.

\section{Conclusions}

Our main conclusions on dwarf starburst galaxies, based on the analyses of several chemical evolution models, are:

- The total number of bursts of star formation can not exceed 10 if one wants to reproduce the observed abundances and gas masses.

- The most important physical parameter in order to explain the observed spread in the chemical properties of these galaxies is the star formation rate. Since we assumed that the star formation law is the same for all the galaxies the difference is contained in the star formation efficiency $\Gamma\left(\mathrm{Gyr}^{-1}\right)$. In order to reproduce the spread observed in the $\log (\mathrm{N} / \mathrm{O})$ vs. $12+\log (\mathrm{O} / \mathrm{H})$, the $\log (\mathrm{C} / \mathrm{O})$ vs. $12+\log (\mathrm{O} / \mathrm{H})$ and $[\mathrm{O} / \mathrm{Fe}]$ vs. $[\mathrm{Fe} / \mathrm{H}]$ planes, the parameter $\Gamma$ should vary from 0.1 to $7 \mathrm{Gyr}^{-1}$ for a normal IMF (either Salpeter (1955) or Scalo (1986)).

- The presence of massive dark matter halos around these galaxies is required in order to avoid their complete destruction due to the starbursts. A ratio $R=\frac{M_{\text {dark }}}{M_{\text {lum }}}=1-50$ is suggested with a concentration $S=\frac{r_{L}}{r_{d}}=0.3-0.4$.

- Enriched galactic winds (enriched by the products of type IISNe) are likely to occur in these galaxies and their efficiency should not exceed $w_{i}=0.1 \mathrm{Gyr}^{-1}$.

- Type II supernovae dominate the energetics and the chemistry of starburst galaxies. On the other hand, from the energetic point of view type Ia SNe and massive stars are negligible.

- However, firm conclusions on the energetics of gas can not yet be drawn since the treatment of the energy transfer from massive stars and supernovae into the ISM is still poorly known. Moreover, a fully dynamical treatment is necessary to assess the existence and the modalities of galactic winds.

Acknowledgments. We are grateful to F. Bradamante, F. Brighenti and M. Tosi for many useful discussions.

\section{References}

Arimoto, N., Yoshii, Y. 1987, A\&A 173, 23

Bertin, G., Saglia, R.P., Stiavelli, M. 1991, ApJ 384, 423

Bradamante, F., Matteucci, F., D'Ercole, A. 1998, A\&A 337, 338

Garnett, D.R, Skillman, E.D., Dufour, R.J. et al. 1995, ApJ 443, 64

Garnett, D.R, Skillman, E.D., Dufour, R.J., Shields, G.A. 1997, ApJ 481, 174

Kobulnicky, H.R., Skillman, E.D. 1996, ApJ 471, 211 
Kumai, Y., Tosa, M. 1992, A\&A 257, 511

Kunth, D., Mas-Hesse, M., Terlevich, E. et al. 1998, A\&A 334, 11

Lequeux, J., Kunth, D., Mas-Hesse, J.M., Sargent, W.L.W. 1995, A\&A 301, 18L

Maeder, A. 1992, A\&A 264, 105

Marconi, G., Matteucci, F., Tosi M. 1994, MNRAS 270, 35

Martin, C.L. 1996, ApJ 465, 680

Matteucci, F. 1986, MNRAS 221, 911

Matteucci, F., Chiosi, C. 1983, A\&A 123, 121

Matteucci, F., Greggio, L., 1986, A\&A 154, 279

Matteucci, F., Tornambé, A. 1987, A\&A 185, 51

Matteucci, F., Tosi, M. 1985, MNRAS 217, 391

Mac Low, M.-M., Ferrara, A. 1998, in: T. Richtler \& J.M. Braun (eds.), The Magellanic Clouds and Other Dwarf Galaxies, Proc. Bonn/Bochum-Graduiertenkolleg (Aachen: Shaker verlag), p. 177

Meurer, C.R., Freeman, K.C., Dopita, M.A., Cacciari, C. 1992, AJ 103, 60

Nomoto, K., Thielemann, F.K., Yokoi, K., 1984, ApJ 286, 644

Pagel, B.E.J., Simonson, E.A., Terlevich, R.J., Edmunds, M.G. 1992, MNRAS 255, 325

Papaderos, P., Fricke, K.J., Thuan, T.X., Loose, H.H. 1994, A\&A 291, 13

Pilyugin, L.S. 1992, A\&A 260, 58

Pilyugin, L.S. 1993, A\&A 277, 42

Renzini, A., Voli, M. 1981, A\&A 94, 175

Salpeter, E.E. 1955, ApJ 121, 161

Scalo, J.M. 1986, Fundam. Cosmic Phys. 11, 1

Skillman, E.D. 1996, in: E.D. Skillman (ed.), Minnesota Lectures on Extragalactic Neutral Hydrogen, ASP-CS 106

Tosi, M., Greggio, L., Focardi, G., Marconi, G. 1992, in: B. Barbuy \& A. Renzini (eds.), The Stellar Populations of Galaxies, Proc. IAU Symp. No. 148 (Dordrecht: Kluwer), p. 207

Thuan, T.X., Isotov, Y.I., Lipovetsky, V.A. 1995, ApJ 445, 108

van den Hoek, L.B., Groenewegen, M.A.T. 1997, A\&AS 123, 305

Weaver, R., McCray, R., Castor, J., Shapiro, P., Moore, R. 1977, ApJ 218, 377

Woosley, S.E. 1987, in Nucleosynthesis and Chemical Evolution, 16th Saas-Fee Advanced Course, Geneva Observatory, p. 1

Woosley, S.E., Weaver, T.A. 1995, ApJS 101, 181

\section{Discussion}

Heckman: (i) In well-studied local starbursts, the physical and dynamical properties of the outflowing gas (super bubbles and super winds) require a much higher efficiency of thermalization of supernova mechanical energy than you have assumed (e.g., of-order unity, rather than a few percent. What would the effect on your model be, if you assumed a higher efficiency?

(ii) Gas-phase $\mathrm{Fe} / \mathrm{O}$ abundance ratios may underestimate the true Fe-abundance, since significant amounts of Fe may be locked in dust-grains.

Matteucci: (1) We tried a higher thermalization efficiency (e.g., of the order of unity) with the consequence of blowing-up the entire system! May be under different conditions and for more massive objects a high thermalization efficiency could be tolerated and 
even be necessary. The problem is the poor physical knowledge of this process. (2) Yes, I agree.

Langer: Your SN Ia rate as a function of time implies a rather short mean lifetime of SN Ia progenitors. What is the underlying progenitor model, and why do not you, as it seems, consider the main sequence star plus the white dwarf progenitor model?

Matteucci: The progenitor model I use is the white dwarf plus a red giant companion. The clock for the explosion is given by the lifetime of the red giant. Since we consider progenitors for the red giant from 0.8 to $8 \mathrm{M}_{\odot}$, the smallest time for the explosion is $3 \times 10^{7}$ years, which is the lifetime of a $8 \mathrm{M}_{\odot}$ star and corresponds to a system of $8 \mathrm{M}_{\odot}+8 \mathrm{M}_{\odot}$.

Van Dyk: (1) Although I have no problem with including what you call 'type Ib supernovae' with the type II supernovae, I would like to point out that type Ib supernovae are members of a larger class of type Ib/type Ic supernovae and out of nearly 50 known examples of SNe Ib/c, only 3 have been well-classified as $\mathrm{SNe} \mathrm{Ib} \mathrm{(the} \mathrm{vast} \mathrm{majority} \mathrm{have}$ been classified as SNe Ic), and only SN1985F in NGC 4618 has even remotely close to what one would expect for an exploding WR star. No solid observational evidence exists that exploding WR stars give rise to SNe b/c; (2) I am happy with your assumption of progenitor ages for the SNe Ia which are younger than the canonical model, but may be too short.

Matteucci: I agree with you on type Ib SNe. There is neither observational nor theoretical evidence to the fact that their progenitors are exploding WR stars. Fortunately, from the chemical evolution point of view it does not make a substantial difference to assume that type Ib SNe originate from exploding WR stars or from binary systems containing white dwarfs but with masses large enough to explode on relatively short timescales. Concerning type Ia SNe, I do not understand what you mean by saying that I do not use the canonical model. I do use the canonical model, namely the system composed by a white dwarf plus a companion with a mass in the range from 0.8 to $8 \mathrm{M}_{\odot}$. In this canonical scenario the fastest system, as I have already said to Dr. Langer, is a $8 \mathrm{M}_{\odot}+8 \mathrm{M}_{\odot}$ system which explodes after 30 million years from its formation. If by canonical scenario you mean the double white dwarf system, then the shortest timescale for the explosion can be longer but not substantially. I think that generally people confuse the time at which the type IaSN rate reaches a maximum $(\sim 1 \mathrm{Gyr}$ for the solar vicinity) with the fastest system appearing.

Legrand: I have a comment about the $\mathrm{C} / \mathrm{O}$ ratio in starburst galaxies. In IZw 18 , the $\mathrm{C} / \mathrm{O}$ ratio is representative of a Salpeter IMF where all the stars have evolved. So this can be well reproduced, both by a previous burst or by a continuous low SFR. Thus I think that the problem is not I Zw 18 but the fact that low metallicity galaxies present a low $\mathrm{C} / \mathrm{O}$. The reason is that to reproduce these low values you need to see the oxygen produced by the most massive stars and not the carbon produced by the intermediate mass stars. This means that you are looking at these objects few Myr after the burst. However, as shown by Kobulnicky in his talk, it seems that the metals produced in a starburts are not immediately visible. So, what we see are the metals produced by a previous star formation event and we can expect that all the stars produced (massive and intermediate) in this past star formation episode have evolved. If so, we would expect a high $\mathrm{C} / \mathrm{O}$ !

Matteucci: Yes, in principle I agree with you that there could be a problem in explaining very low $\mathrm{C} / \mathrm{O}$ ratios, but we need to be more quantitative and specify exactly the value of the $\mathrm{C} / \mathrm{O}$ ratio. A variable or different than a Salpeter-like IMF (for example an IMF biased towards massive stars) could perhaps solve the problem. 